\title{
STAT1/3 and ERK1/2 Synergistically Regulate Cardiac Fibrosis Induced by High Glucose
}

\author{
Bin Dai ${ }^{a}$ Meng Cui ${ }^{b} \quad$ Mei Zhu ${ }^{a}$ Wen-Ling Su ${ }^{a} \quad$ Ming-Cai Qiu ${ }^{a}$ Hong Zhang ${ }^{c}$ \\ aDepartment of Endocrinology, Tianjin Medical University General Hospital, Tianjin Heping District,

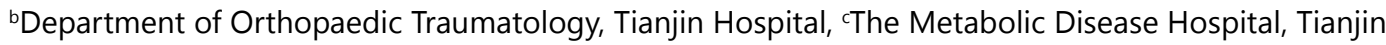 \\ Medical University, Tianjin, P. R. China
}

\section{Key Words}

High glucose - Hyperglycemia - Diabetes - STAT1 - STAT3 - ERK1/2 - Cardiac fibroblast • Collagens I and III • Cardiac fibrosis • S31-201 • Fludarabine

\begin{abstract}
Background: Hyperglycaemia promotes the proliferation of cardiac fibroblasts (CFs) and collagen synthesis in CFs. However, the molecular mechanism underlying the effects of HG on proliferation and collagen synthesis of $\mathrm{CF}$, is not completely understood. Objectives: The objectives of the present study were to determine whether the STAT proteins has a functional role in high glucose-induced proliferation of CFs and collagen synthesis in vitro and whether the STAT signaling pathway and MAPK signaling pathway have synergetical effects on high glucose-mediated cardiac fibroblasts proliferation and collagen synthesis. Methods: Rat CFs were cultured in Dulbecco's modified Eagle's medium, supplemented with 5.5 or $25 \mathrm{mmoh}$ D-glucose, in the presence of absence of STAT1 inhibitor Fludarabine, STAT3 inhibitor S31-201 and ERK1/2 inhibitor PD98059. Proliferation were measured by the 3-(4,5-dimethyl-2 thiazoyl)2,5-diphenyl-2H-tetrazolium bromide (MTT) assay, the production of Type I and III collagen was evaluated using real-time quantitative RT-PCR and ELISA, and the phosphorylation expression of STAT1 and STAT3 were analyzed by Western blot. Results: High glucose treatment promoted the proliferation of cardiac fibroblasts and collagen types I and III synthesis. High glucose treatment induced STAT1 and STAT3 phosphorylation in cardiac fibroblasts, the mode and level of STAT1 and STAT3 phosphorylation were significantly different. Fludarabine and S31-201 could both inhibited high glucose stimulated proliferation of cardiac fibroblasts and collagen types I and III synthesis with different effects. Combination of Fludarabine and PD98059 or combination of S31-201 and PD98059 both exhibited stronger inhibitions on proliferation of cardiac fibroblasts and collagen types I synthesis, but the effects and functional modes are different. Conclusion: Both STAT1 and STAT3 mediate the proliferation of cardiac fibroblasts and collagen synthesis induced by high glucose. STAT1 and STAT3 both have synergetic effects with ERK1/2 on regulating proliferation of cardiac fibroblasts and collagen types I synthesis.
\end{abstract}

Copyright $(2013$ S. Karger AG, Basel 


\section{Introduction}

Long-term diabetes can result in the development of diabetic cardiomyopathy independent of hypertension and coronary heart disease. Heart failure due to diastolic ventricular dysfunction is a characteristic of diabetic cardiomyopathy and can occur during the early stages of diabetes [1]. Cardiac fibrosis is one of the major pathological processes of diabetic cardiomyopathy [2] and manifests as enhanced proliferation of cardiac fibroblasts (CFs) and excessive deposition of extracellular matrix (ECM), such as collagens. Hyperglycemia is one of the major pathological manifestations of diabetes and may promote the development of heart failure, primarily by causing excessive accumulation of collagen within the interstices of the myocardium, which can result in impaired diastolic and systolic functions $[3,4]$. Treatment of cultured CFs with high concentrations of glucose (HG) results in increased proliferation of CFs and collagen synthesis [5-7]. However, the molecular mechanisms underlying the effects of $\mathrm{HG}$ on $\mathrm{CF}$ function, including proliferation and collagen synthesis, are not completely understood.

STATs belong to an intracellular transcription factor family that primarily mediate cytokine- and growth factor-induced signals that are activated by diverse biological responses including proliferation, differentiation and apoptosis [8]. The STAT signaling pathway has been shown to be an integral part of the responses of the myocardium to various cardiac insults, including myocardial infarction, oxidative damage, myocarditis, hypertrophy and remodeling, in addition to having a prominent role in cardioprotective therapies such as ischemic preconditioning [9]. The STAT pathway also has been documented to take a part in the pathogenesis of liver fibrosis, pulmonary fibrosis and renal fibrosis [10-12], as an especially important mechanism for renal fibrosis by which hyperglycemia contributes to renal damage [13]. These results predict that STAT may have a role in the process of myocardial fibrosis induced by HG. To date, the majority of data involving STAT activity in the heart has been focused on STAT1 and STAT3.

The ERK1/2 signaling pathway has long been recognized as the intracellular signal transduction enzymes which are critically involved in regulating proliferation, differentiation and apoptosis. ERK1/2 also participate in the apoptosis caused by the cardiac reperfusion injury [14]. HG promotes expression of collagen I and III via activating ERK1/2, and inhibition of ERK1/2 phosphorylation significantly decreases the mRNA and protein levels of collagen I and III [15]. Within the intracellular signal transduction networks, both ERK1/2 and STAT are downstream proteins of gp130. STAT protein sequences contains highly conserved ERK phosphorylation sites; and therefore STAT protein can be activated by ERK [16-19]. The cross-talk between these two signaling cascades has been shown to participate in a series of physiological and pathological processes [20,21]. Hence the ERK1/2 mediated-synthesis of collagen I and III is likely involved in the activation of STAT3.

Based on these results, we hypothesized that HG stimulates STAT phosphorylation via activating ERK1/2, and ERK1/2 and STAT cooperatively promote collagen synthesis in CFs through a crosstalk mechanism. The present study was designed to test this hypothesis by investigating whether the STAT and MAPK signaling pathways have synergetic effects on HGinduced proliferation and collagen synthesis in CFs.

\section{Materials and Methods}

\section{Materials and reagents}

Wistar rats were obtained from the Laboratory Animal Centre of Tianjin Medical University. 3-(4,5-dimethyl-2 thiazoyl)-2,5-diphenyl-2H-tetrazolium bromide (MTT), trypsin and Dulbecco's modified Eagle's medium (DMEM) were purchased from Sigma-Aldrich (St Louis, MO, USA). Fludarabine (STAT1 inhibitor), s31-201 (STAT3 inhibitor), PD98059 (ERK1/2 inhibitor) were purchased from Calbiochem (La Jolla, CA). Trizol reagent was obtained from Invitrogen (Carlsbad, CA). Reagents for real-time RT-PCR were purchased from TaKaRa (Tokyo, Japan). Rabbit phospho-specific monoclonal antibodies for STAT1 and 
Dai et al.: Signaling Mechanisms for High Glucose-Induced Cardiac Fibrosis

STAT3 and rabbit monoclonal antibodies against total STAT1 and STAT3 were purchased from Cell Signaling Technology (Danves, MA). Horseradish peroxidase (HRP)-conjugated goat anti-rabbit IgG was obtained from KPL (Gaithersburg, MD, USA). The bicinchoninic acid (BCA) Protein Assay kit was obtained from Pierce (Rockford, IL, USA). The chemiluminescence (ECL) kit was obtained from Perkin Elmer (Massachusetts, USA). Reagents for ELISA analysis of collagen types I and III were purchased from Bionewtrans Pharmaceutical Biotechnology (Franklin, USA). All chemicals were of the highest possible purity grade.

\section{Cell culture}

Cardiac fibroblasts were isolated from 1- to 3-day-old Wistar rats of either gender. Animal experiments were conducted in accordance with the guidelines established by the University of Tianjin Animal Care and Use Committee, who provided approval for these experiments. In brief, rats were sacrificed by cranial dislocation, and the hearts were removed quickly under sterile conditions. Ventricular tissue was excised, minced, and digested with $0.25 \%$ (w/v) trypsin in Hank's buffer. The CF suspension was plated onto tissueculture dishes for 30 min then non-adherent cells were washed away. Cells were maintained in DMEM containing $5.5 \mathrm{mM}$ glucose supplemented with $10 \%$ fetal calf serum and $1 \%$ penicillin and streptomycin at $37^{\circ} \mathrm{C}$ in a humidified incubator under $5 \% \mathrm{CO}_{2}$. Confluent cells were passaged with $0.05 \%$ trypsin and plated directly onto $3.8 \mathrm{~cm}^{2}$ wells of a 12-well plate filled with $2 \mathrm{ml}$ culture medium. CFs were identified and characterized by immunophenotyping for vimentin. CFs of the second to the fourth passages were used for our experiments.

\section{Experimental protocols}

$5.5 \mathrm{mM}$ or $25 \mathrm{mM}$ glucose concentration was used in this study. After incubation in DMEM containing $5.5 \mathrm{mM}$ glucose with $10 \% \mathrm{FCS}$, the CFs were exposed to DMEM containing $5.5 \mathrm{mM}$ glucose for $24 \mathrm{~h}$. The CFs were pre-incubated in the presence or absence of fludarabine $(50 \mu \mathrm{M})[22], \mathrm{S} 31-201(10 \mu \mathrm{M})$ [23], PD98059 $(10 \mu \mathrm{M})[15]$ for $2 \mathrm{~h}, 45 \mathrm{~min}$ and $30 \mathrm{~min}$ respectively. They were then further incubated with DMEM containing $5.5 \mathrm{mM}$ glucose (normal glucose), $5.5 \mathrm{mM}$ glucose $+19.5 \mathrm{mM}$ mannose (osmotic control), or 25 $\mathrm{mM}$ glucose (high glucose), at $37^{\circ} \mathrm{C}$ for varying periods $(12,24,48$ and $72 \mathrm{~h})$. Finally, the cells were harvested for real-time PCR. The medium was collected and stored at $-80^{\circ} \mathrm{C}$ for enzyme linked immunosorbent assay (ELISA). Cells were harvested for Western blotting after co-incubation at $37^{\circ} \mathrm{C}$ for $0,30,60$, and $120 \mathrm{~min}$. All measurements were repeated three times.

\section{Cell proliferation assay}

Cell proliferation was evaluated by MTT assay. Briefly, CFs were seeded in 96 -well culture plates $\left(5 \times 10^{3}\right.$ cells/well) with DMEM containing 10\% FCS and $5.5 \mathrm{mM}$ glucose. Then they were replaced with DMEM containing $5.5 \mathrm{mM}$ glucose for $24 \mathrm{~h}$. Depending on the experimental group, CFs were pre-incubated in the presence or absence of fludarabine $(50 \mu \mathrm{M})$, S31-201 $(10 \mu \mathrm{M})$, or PD98059 $(10 \mu \mathrm{M})$ for $2 \mathrm{~h}, 45 \mathrm{~min}, 30 \mathrm{~min}$, respectively. The medium was then replaced with DMEM containing $5.5 \mathrm{mM}$ glucose (normal glucose), 5.5 mM glucose plus $19.4 \mathrm{mM}$ mannose (osmotic control), or $25 \mathrm{mM}$ glucose (high glucose). Next, the CFs were further incubated for $24 \mathrm{~h}$ and $48 \mathrm{~h}$ in the presence or absence of fludarabine, S31-201, or PD98059. Four hours before the end of the experiment, $20 \mu \mathrm{L}$ of $5 \mathrm{mg} / \mathrm{mL}$ MTT was added to each well. After $4 \mathrm{~h}$, the culture medium was removed and $100 \mu \mathrm{L}$ dimethylsulphoxide (DMSO) was added to each well. The absorbance for each well was determined at $490 \mathrm{~nm}$. Blank control wells were designed without cells, but with the addition of culture medium in parallel to the experimental wells.

\section{Quantitative real-time RT-PCR}

Total RNA sample was extracted using Trizol reagent according to the manufacturer's procedures. Five micrograms of total RNA was reverse transcribed with Promega MMLV reverse transcriptase and relevant reagents. The resultant first-strand DNA $(0.5 \mu \mathrm{l})$ was amplified with SYBR® Premix Ex TaqTM. The mRNA expression of $\beta$-actin and procollagen types I and III were quantified. The test mRNAs were all normalized to GAPDH mRNA. Specific primers for GAPDH were: Forward primer 5'-GGG GTG ATG CTG GTG CTG AG-3', Reverse primer 5'-GAT GCA GGG ATG ATG TTC TG-3', amplified product $370 \mathrm{bp}$; for $\alpha$ I procollagen types I: Forward primer 5'-AGC CAC CAG CCC CTC ACT-3', Reverse primer 5'-CGA GGT AGT CTT TCA GCA ACA CAG T-3', amplified product 160 bp; For $\alpha$ I procollagen types III: Forward primer 5'-CAA CAC CGA TGA GAT TAT G-3', Reverse primer 5'-TCA GGA TTG CCG TAG C-3', amplified product $344 \mathrm{bp}$. The amplification conditions 
Fig. 1. Real-time RT-PCR analysis of mRNA expression of collagen type I (A) and III (B) in rat cardiac fibroblasts (CFs). CFs were treated with DMEM and $5.5 \mathrm{mM}$ glucose (NG), 5.5 $\mathrm{mM}$ glucose plus $19.5 \mathrm{mM}$ mannose (OC), or $25 \mathrm{mM}$ glucose (HG) in the presence or absence of STAT1 inhibitor fludarabine (FLU; $50 \mu \mathrm{M})$, STAT3 inhibitor S31-201 (S31; $10 \mu \mathrm{M})$, or ERK1/2 inhibitor PD98059 (PD; $10 \mu \mathrm{M})$. Values are the mean \pm SD of three measurements. ${ }^{*} P<0.01$ and ${ }^{* *} P<0.001$ for comparisons NG and OC with HG; ${ }^{\#} P<0.01$ and ${ }^{\# \#} P<0.001$ were used to compare inhibitors with HG alone.

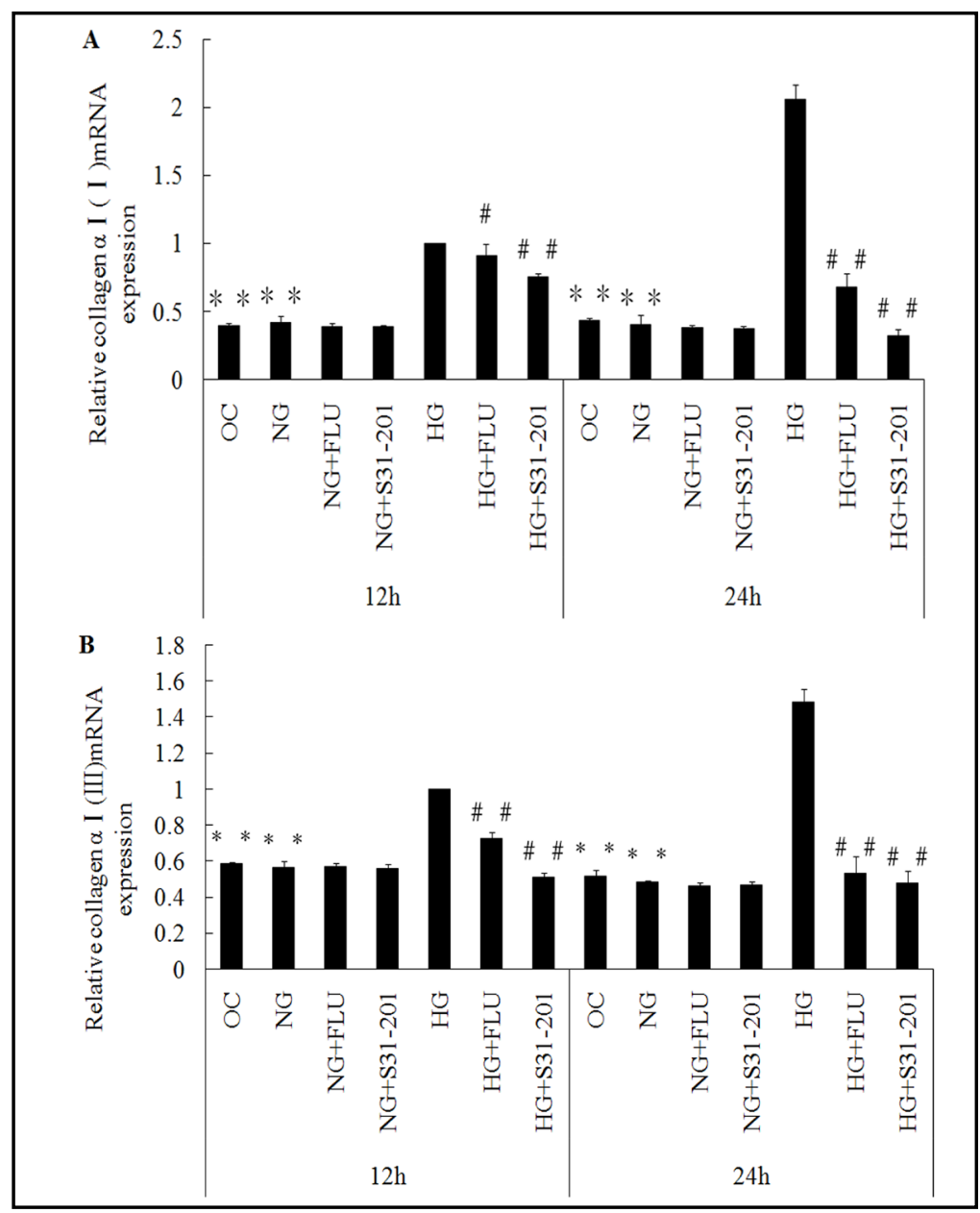

were, predenaturation at $94^{\circ} \mathrm{C}$ for $4 \mathrm{~min}$, denaturation at $94^{\circ} \mathrm{C}$ for $30 \mathrm{~s}$, annealing at $50^{\circ} \mathrm{C}$ for $30 \mathrm{~s}$ and extension at $72^{\circ} \mathrm{C}$ for $40 \mathrm{~s}$ for 40 cycles. The relative changes in gene expression were analyzed with the 2(-Delta C(T)) method [24]. Specificity of RT-PCR was checked by analyzing melting curves and by gelelectrophoresis of the amplicons.

\section{Enzyme-linked immunosorbent assay (ELISA)}

Soluble collagen I and III proteins were determined by ELISA kit according to the manufacturer's protocols, which detects the secretion of procollagen I C-terminal peptide (PICP) and procollagen III $\mathrm{N}$-terminal peptide (PIIICP).

\section{Western blot analysis}

After rinsing with cold D-Hanks buffer, CFs were collected and lysed. Protein samples were extracted and measured using a BCA Protein Assay kit. Approximately $32 \mu \mathrm{l}$ protein in $8 \mu \mathrm{l} 5 \times$ Loading Buffer were separated by $10 \%$ sodium dodecyl sulphate-polyacrylamide gel electrophoresis (SDS-PAGE) and transferred to polyvinylidene fluoride (PVDF) membranes (350 mA, $120 \mathrm{~min}$ ). Membranes were blocked with $5 \%$ fat-free milk in TBST buffer (20 mM Tris- $\mathrm{HCl}, \mathrm{pH} 7.5 ; 150 \mathrm{mM} \mathrm{NaCl}$ and $0.05 \%$ Tween 20 ) and were subsequently incubated with primary antibodies (anti-STAT1, 1:500; anti-STAT3, 1:500; anti-pSTAT1, 1:500; and anti-p-STAT3, 1:500) at $4^{\circ} \mathrm{C}$ overnight. Then, the membranes were washed with TBST and incubated for $2 \mathrm{~h}$ with HRP-conjugated secondary antibodies. The membranes were finally developed using an electrochemiluminescence (ECL) kit. Band intensity was quantified by gel densitometry with the Gel Image Analysis System (UVP). Protein phosphorylation was normalized against total protein band densitometry on an individual basis. 
Fig. 2. Effects of high glucose (HG) on production of collagen type I (A) and III (B) in rat cardiac fibroblasts (CFs), determined by ELISA. CFs were treated with DMEM and 5.5 $\mathrm{mM}$ glucose (NG), $5.5 \mathrm{mM}$ glucose plus $19.5 \mathrm{mM}$ mannose (OC), or 25 $\mathrm{mM}$ glucose $(\mathrm{HG})$ in the presence or absence of STAT1 inhibitor fludarabine (FLU; $50 \mu \mathrm{M}$ ), STAT3 inhibitor S31-201 (S31; $10 \mu \mathrm{M})$, or ERK1/2 inhibitor PD98059 (PD; $10 \mu \mathrm{M}$ ). Values are the mean \pm SD of three measurements. ${ }^{*} P<0.01$ and ${ }^{* *} P<$ 0.001 for comparisons NG and OC with HG; ${ }^{\#} P<0.01$ and ${ }^{\# \#} P<0.001$ were used to compare inhibitors with HG alone.

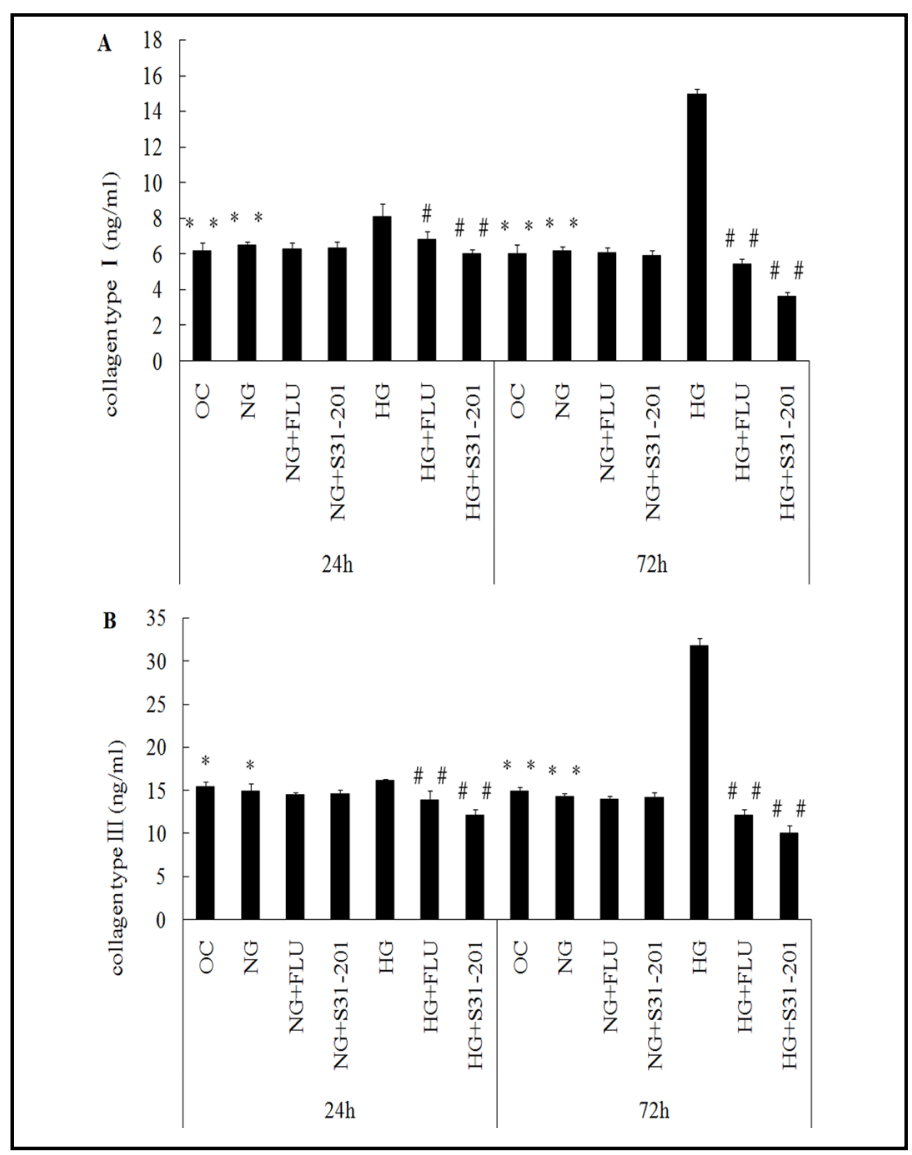

\section{Statistical analysis}

All values are shown as means \pm SD. Differences between two groups were determined with unpaired Student's $t$-test. ANOVA was used for multiple comparisons. A $P<0.05$ was considered statistically significant.

\section{Results}

Enhancement of proliferation and collagen synthesis in cardiac fibroblasts by high glucose

Confluent cardiac fibroblasts treated with HG showed a significant increase in proliferation at $24 \mathrm{~h}$ to $48 \mathrm{~h}$ (Fig. $4 \mathrm{~A}$ ). The mRNA levels of procollagens I and III were upregulated from $12 \mathrm{~h}$ to $24 \mathrm{~h}$ after incubation with HG (Fig. 1A \& B). Consistently, the protein levels of collagens I and III in the supernatant were also increased, but this upregulation occurred at later time points $(24 \mathrm{~h}$ to $72 \mathrm{~h}$ ). These effects were not observed with highosmolarity treatment, which ruled out osmotic pressure changes as being responsible for the increased level of collagens (Fig. 2A \& B).

Enhancement of STAT1 and STAT3 activities in cardiac fibroblasts by high glucose

The activity of STAT1 (the phosphorylated form of STAT1) was not changed in CFs exposed to HG within 0-60 min, but was significantly increased between 90 and $120 \mathrm{~min}$ in HG. By comparison, the activity of STAT3 was decreased within 0-30 min in HG culture, followed by significant increases between 90 and $120 \mathrm{~min}$. Moreover, the increase of STAT3 activity was remarkably greater than that of STAT1 (2.83 fold $v s 1.94$ fold; $P<0.05$; Fig. 3). 
Fig. 3. Effects of high glucose (HG, $25 \mathrm{mM}$ ) on STAT1 and STAT3 activities at varying time points. STAT1 and STAT3 activities were determined by Western blot analysis of the protein levels of phosphorylated forms of STAT1 (p-STAT1) and STAT3 (p-STAT3), normalized to total STAT1 and STAT3 levels. (A) Representative Western blot bands from three experiments. (B) Quantitative results of p-STAT1 and p-STAT3. The results were normalized by arbitrarily setting the densitometry of vehicle-treated cells ( $0 \mathrm{~min}$ ) to 1.0. Values are the mean \pm SD of three measurements. ${ }^{\#} P<0.01$ and ${ }^{\# \#} P<0.001$ vs control.

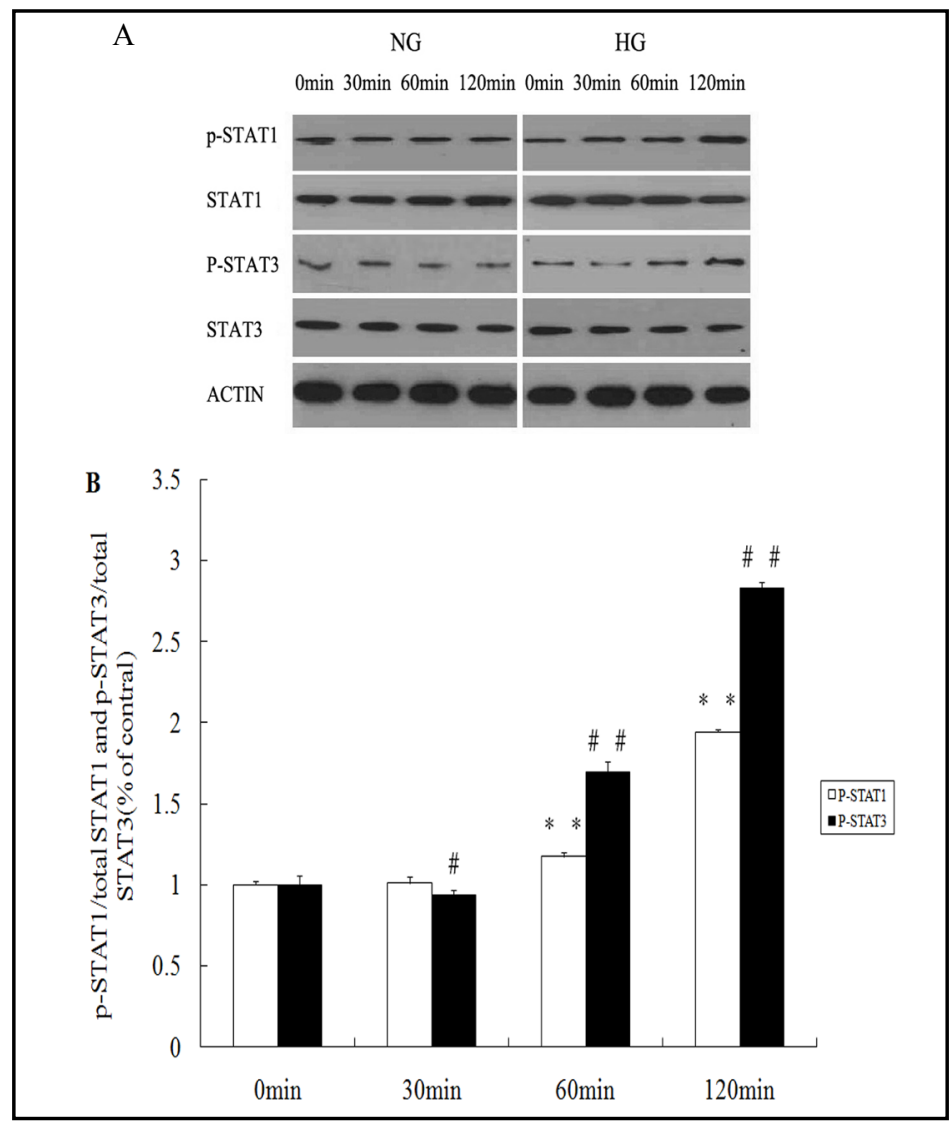

Suppressing effects of STAT1 and STAT3 inhibition on high glucose-induced CF proliferation and collagen synthesis

To elucidate HG-mediated signaling pathways, CFs were pretreated with STAT1 inhibitor fludarabine and STAT3 inhibitor S31-201. As illustrated in Figure 4B, these inhibitors substantially suppressed the HG-stimulated CFs proliferation $24 \mathrm{~h}$ after treatment. Furthermore, fludarabine and S31-201 also significantly reversed the HG-induced upregulation of procollagens I and III mRNAs from 12 to $24 \mathrm{~h}$ after drug treatment (Fig. $1 \mathrm{~A}$ \& B). Fludarabine and S31-201 both significantly suppressed the HG-induced production of collagens I and III after $24 \mathrm{~h}$ treatment (Fig. 2A \& B). Moreover, at $12 \mathrm{~h}$ after treatment, fludarabine and S31-201 both exhibited stronger inhibitory effects on procollagen III mRNA than procollagen I (fludarabine: $27.7 \%$ for procollagen I vs $8.1 \%$ for procollagen III, $P<0.05$; S31-201: $48.9 \%$ for procollagen I vs $24.3 \%$ for procollagen III, $P<0.05)$. In contrast, at 24 $\mathrm{h}$, the effects of S31-201 were smaller for procollagen I than for procollagen III $(67.7 \% \mathrm{vs}$ $84.4 \%, P<0.05$ ).

Synergetic effects of combined inhibition of STAT1 or STAT3 and ERK1/2 on high glucoseinduced CF proliferation and collagen synthesis

PD98059 suppressed the HG-stimulated proliferation of CFs (Fig. 4A) and attenuated the HG-induced production of collagens I/III (Fig. 5A \& B). The combination of fludarabine and PD98059 produced stronger inhibition of CF proliferation at $48 \mathrm{~h}$ and collagen I production at $72 \mathrm{~h}$, compared with either fludarabine or PD98059 alone (Fig. 4B \& Fig. 5A). Similarly, co-treatment of CFs with S31-201 and PD98059 exhibited stronger inhibitory effects on proliferation and collagen I production at $24 \mathrm{~h}$ and $48 \mathrm{~h}$, compared with either S31-201 or PD98059 alone (Fig. 4B \& Fig. 5A). The effects on HG-stimulated collagen III synthesis were not different between any combinational drug treatment or any single drug treatment (Fig. 5B). 
Fig. 4. MTT assay on the effects of high glucose (HG) on cardiac fibroblast (CF) proliferation. CFs were treated with DMEM and $5.5 \mathrm{mM}$ glucose (NG), $5.5 \mathrm{mM}$ glucose plus $19.5 \mathrm{mM}$ mannose (OC), or $25 \mathrm{mM}$ glucose (HG) in the presence or absence of STAT1 inhibitor fludarabine (FLU; $50 \mu \mathrm{M}$ ), STAT3 inhibitor S31-201 (S31; $10 \mu \mathrm{M})$, or ERK1/2 inhibitor PD98059 (PD; $10 \mu \mathrm{M})$. Values are the mean \pm SD of three measurements. ${ }^{*} P<0.01$ and ${ }^{* *} P$ $<0.001$ for comparisons NG and $\mathrm{OC}$ with $\mathrm{HG}$; $P<0.01$ and ${ }^{\# \# P} P<0.001$ were used to compare inhibitors with HG alone.

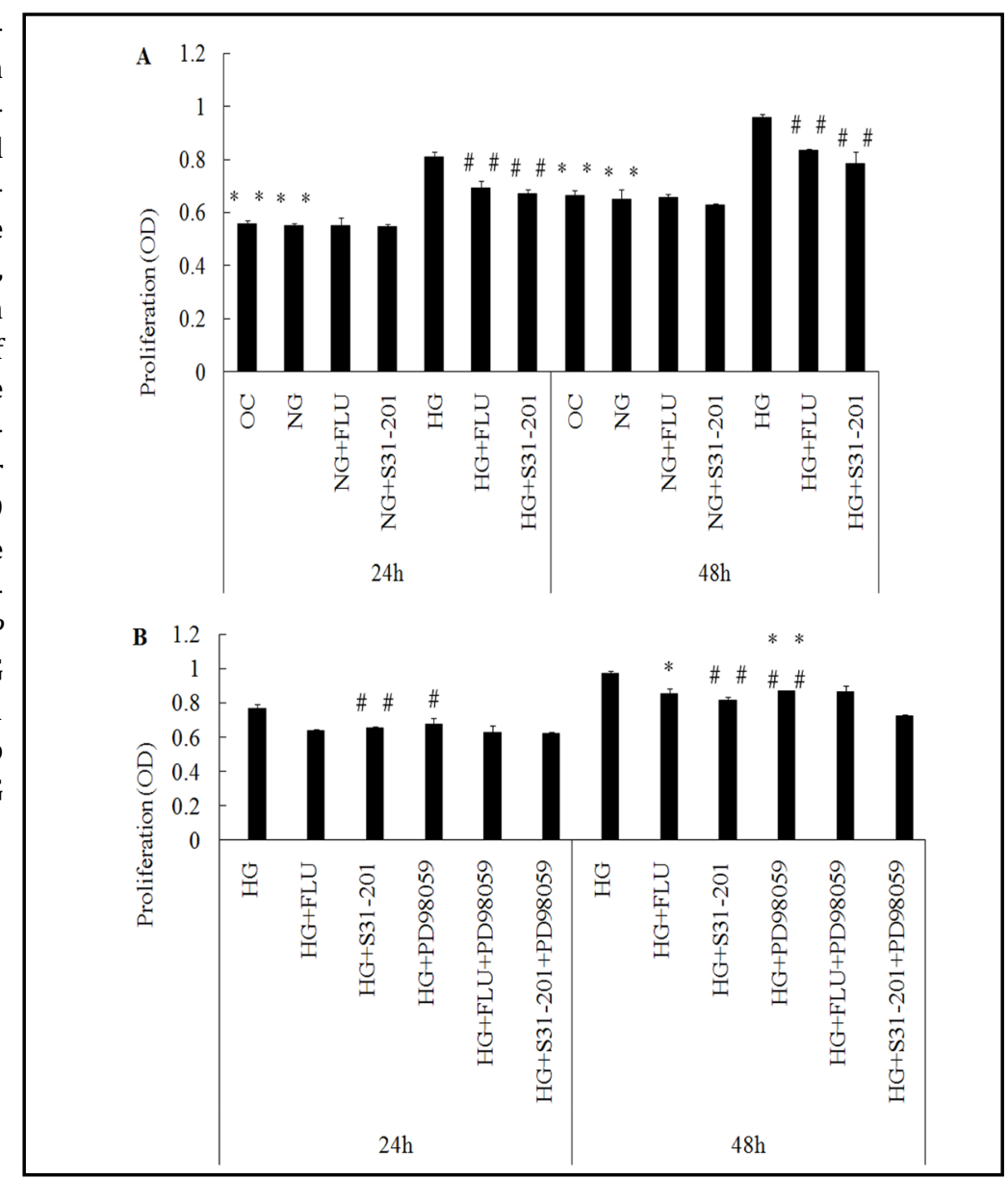

\section{Discussion}

In the present study, we investigated the effects of high extracellular glucose on the proliferation of CFs and synthesis of type I and type III collagens in rat CFs. We found that HG promoted CF proliferation and collagens I/III synthesis. There are several new findings in the present study: (1) Both STAT1 and STAT3 were activated by HG in CFs, and this activation promoted HG-induced CF proliferation and collagen synthesis; (2) HG-stimulated CF proliferation and collagen production could be reversed by inhibition of STAT1/3 activation. To our knowledge, this is the first report showing the involvement of STATs in HG-induced proliferation and collagen synthesis in CFs; And (3) combined inhibition of STAT1 and ERK1/2 or of STAT3 and ERK1/2 using specific pharmacological probes both produced significantly stronger suppression of CF proliferation and collagen I synthesis, compared with individual inhibition of these signaling factors. These data indicated that there is a synergetic effect between STAT1/3 and ERK1/2 on cardiac fibrogenesis. Based on these findings, we concluded that in addition to ERK1/2, which has been shown contribute to cardiac fibrosis, STAT1/3 also play an important role in regulating this process and their effects are reversible, indicating that the STAT signaling pathway may be involved in the adverse structural remodeling associated with cardiac fibrogenesis. Therefore, these molecules may be new targets for the treatment of pathological processes related to HG, particularly the pathogenesis of diabetic cardiomyopathy, but ascribe to the limitation of animal experiments, the $25 \mathrm{mM}$ high glucose concentration for incubation which was 5 time higher than the normal glucose is rarely reached in the human blood. A protocol requiring lower glucose concentration for longer period would be helpful for understanding the importance of the findings of the current studies in future. 
Fig. 5. ELISA analysis of the effects of combinational inhibition of STAT1 by fludarabine (FLU) and ERK1/2 by PD98059 or of STAT3 by S31201 and ERK1/2 by PD98059 on the production of collagen types I (A) and III (B) in cardiac fibroblasts (CFs) induced by high glucose (HG). Values are the mean \pm SD of three measurements. ${ }^{*} P<0.01$ and ${ }^{* *} P<$ 0.001 for comparisons of FLU or PD98059 with their combination; ${ }^{\#} P<0.05$ for comparisons of S31201 or PD98059 with their combination.
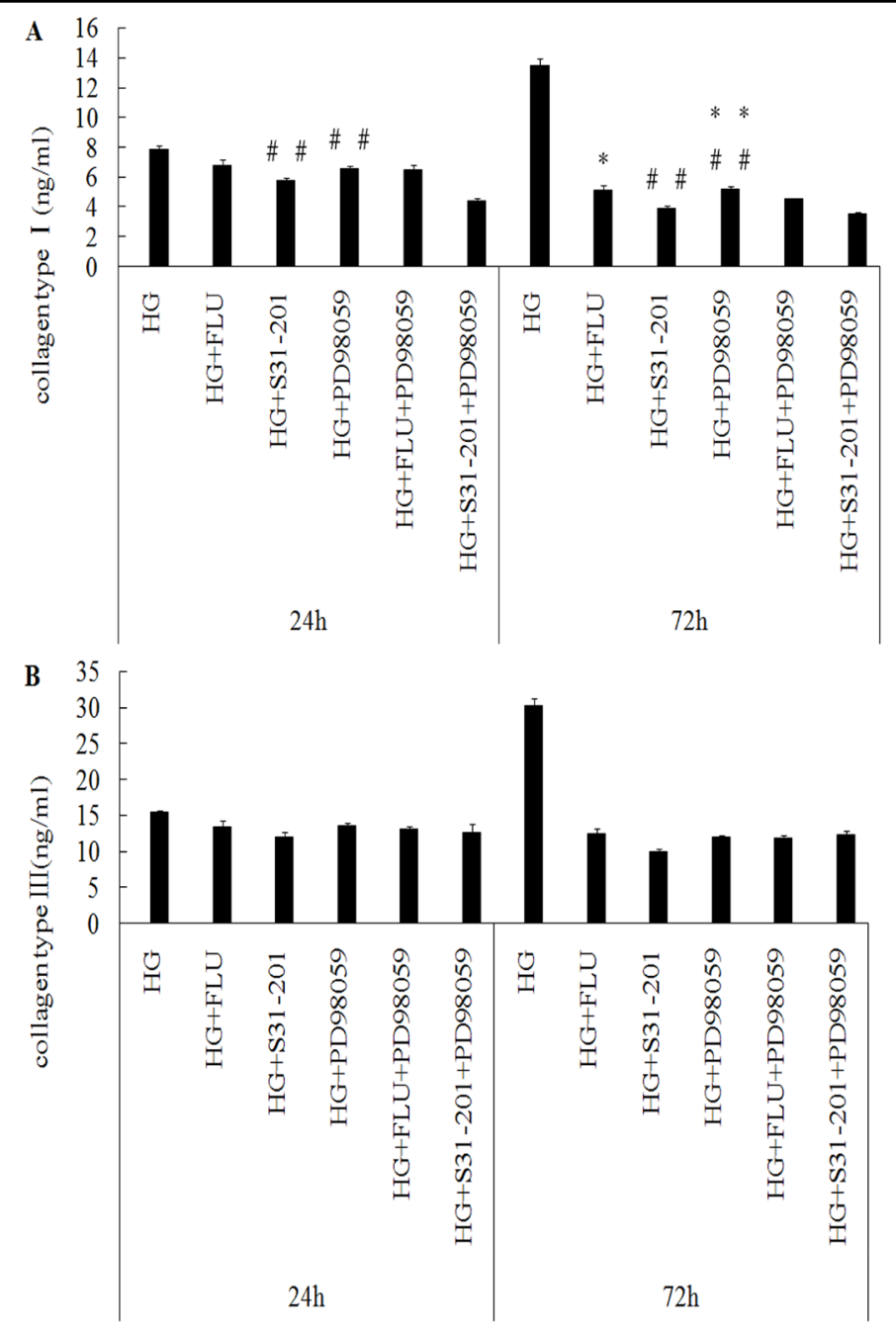

Myocardial fibrosis is a common pathological mechanism for a variety of cardiovascular diseases. Cardiac fibroblasts, important participants in the development of adverse myocardial fibrosis of the heart, are the predominant secretary cell types located within the extracellular matrix (ECM) $[25,26]$. Cardiac fibroblasts take a part in the maintenance of myocardial function mainly by producing the type I and type III collagens and by secreting growth factors [27]. Prolonged activation of CFs, defined by increased proliferation and a subsequent increase of collagen deposition in the ECM, can lead to cardiac fibrosis, which is involved in the pathogenesis of diabetic cardiomyopathy $[2,28]$. In our study, we found that HG promoted proliferation of CFs and synthesis of type I and type III collagens in CFs. These findings are in agreement with the results from other studies[5, 7, 29] and further support the stimulating effect of HG on cardiac fibrosis.

The STAT family consists of seven members: STAT1, STAT2, STAT3, STAT4, STAT5a, STAT5b and STAT6 which are structurally similar proteins and can be activated by their upstream proteins-JAKs [30]. STAT1 and STAT3 are phosphorylated by JAK at tyrosine 701 and tyrosine 705, respectively, which promotes their nuclear translocation. Moreover, they can also undergo serine phosphorylation at position 727 which is cell- and stimulus-specific. Previous studies have shown that activation of STAT1 and STAT3 in the heart plays a very significant role in cardiac pathophysiology during various diseases caused by mechanical overload, ischemia, viral infection, etc [31-33]. In our study, we observed that both STAT1 
and STAT3 were activated by HG to mimic hyperglycemia in diabetes, and this activation was accompanied by enhanced CF proliferation and collagen production. Moreover, we found that exposure of CFs to fludarabine and S31-201 inhibited STAT1 and STAT3, respectively, and resulted in diminished phosphorylation of STAT1 and STAT3, along with decreased CF proliferation and collagen synthesis. Clearly, activation of STAT1/3 is involved in cardiac fibrogenesis, a process that can be reversed by inhibition of STAT1/3 activities.

However, there are two studies on the effect of a high-glucose environment on STATs are not in agreement with our findings.Aberrant regulation of macrophages has been implicated in the onset and progression of type 1 diabetes (T1D) . Macrophages from nonobese diabetic(NOD) mouse, an animal model of T1D, entail developmental and functional defects that are often associated with hypo-responsiveness to interferon (IFN)- $\gamma$. This hyporesponsiveness is partly ascribe to the downstream STAT1 pathway of intracellular signaling being selectively inhibited and the subsequent DNA binding activity being impaired in NOD macrophages exposed to IFN- $\gamma$ [34].Previously, it has been reported that the hBD2 promoter contains binding sites for STAT-1 [35]. High-glucose cultivated keratinocytes expressed reduced phosphorylated signal transducer and activator of transcription (pSTAT)-1 and levels of hBD2 subsequently. The inadequate expression of hBD2 during hyperglycemia condition contributes to impaired wound healing in diabetic patients [36]. These studies indicated that STATs signalling have multifaced role during the pathogenesis development of diabetic.Under the different extracellar stimutions and intracellar environments, the increasing or decreasing expression of STATs mediate different biological effects which related to the causative agents and mechanisms underlying the pathogenesis of diabetic.

Hyperglycemia-mediated proliferation of cardiac fibroblasts and synthesis of collagens are cooperatively regulated by many intracellular and extracellular factors [37]. In addition to the STAT pathway, HG can also promote the production of collagens via activation of ERK1/2 [15]. A few studies have reported that there is crosstalk between ERK1/2 and STAT1/3 during the process of intracellular inflammatory signal transduction [16] and this crosstalk is dependent on the phosphorylation of the S727 on STAT1 and STAT3 [38]. Furthermore, it is commonly accepted that myocardial fibrosis is a chronic inflammatory process [39]. These findings led us speculate that there is an interaction between ERK1/2 and STAT1/3 in HGinduced CF proliferation and collagen I synthesis. This notion was indeed supported by our experiments comparing the combinational pharmacological inhibition of both ERK1/2 and STAT1 or STAT3 and individual inhibition of these signaling factors separately, which clearly demonstrated a synergetic effect between the two distinct signaling pathways. Intriguingly, no such a crosstalk was found for the HG-induced production of collagen III in CFs.

As for the molecular mechanism of synergistic activation of STATs and ERK 1/2 signaling pathway, A few studies provide the insights for us to speculate.The onset and progression of IDDM are under the control of both T helper 1(Th1) and Th2 cells and their respective cytokines [40, 41].The T helper (Th) 2 cytokine IL-31 induces pro-inflammatory effects in activated human macrophages via STAT1 and 5 phosphorylation, and Interleukin31-induced ERK 1/2 activation contributes to the underlying mechanism of Th1 cytokine IL-12 suppression in macrophages which be relevant in Th2 inflammatory responses [42], in addition, the inflammatory effects of IL-31 in human primary keratinocytes (HPKs) can be fulfiled via activating STAT3 phosphorylation in HPKs[43]. These studies showed that T-cell cytokine IL-31 can activating both STATs and ERK 1/2 signaling pathway to take part in the inflammatory lesion course of IDDM. Myocardial tissue inflammatory lesions such as excessive activation of reactive oxygen systems, inflammatory cell infiltration, abnormally high levels of inflammatory cytokines, and so on, can stimulate increasing collagen synthesis in heart and lead to myocardial fibrosis eventually [44].So,it is suggested that activation of both STATs and ERK 1/2 signaling pathway during HG-induced cardic fibrosis probably be mediated by T-cell cytokines such as: IL-31.

As the members of STAT family, STAT1 and STAT3 are structurally similar proteins, but they are functionally heterogeneous [30]. Myocardial damage can induce expression of STAT1 and STAT3 through cytokines, growth factors and reactive oxygen species [45]. Their 
target genes are upregulated by the STAT pathway following cardiovascular injury. STAT1 is a transcription factor responsible for transactivation of genes involved in apoptosis, STAT3 accounts for transactivation of genes involved in survival, hypertrophy and angiogenesis [46-49]. As for the roles of STAT1 and STAT3 in cardiac fibroblasts, a previous study showed that STAT1 and STAT3 have differential effects on cardiac fibrosis [44]. It has been reported that cultured cardiac fibroblasts treated with IL-6 showed significant increases in collagen expression and phosphorylation of STAT1 and STAT3, and inhibition of either STAT1 or STAT3 decreased collagen expression. In the case of STAT3, reduced phosphorylation was believed to underlie the effect on collagen expression, but for STAT1, inhibition of phosphorylation does not decrease collagen expression [50]. In our study, STAT1 and STAT3 both can regulate the HG-induced proliferation of CFs and collagen synthesis, which is inconsistent with the results obtained from the IL- 6 study. Yet there are some important differences between STAT1 and STAT3. Firstly, although STAT1 and STAT3 could both be activated by HG, STAT3 phosphorylation was decreased at early stage but increased later on after exposure to HG. And the enhancement of STAT3 phosphorylation was more obvious compared to STAT1. Secondly, inhibition of STAT1 exhibited a stronger inhibitory effect on HG-induced expression upregulation of procollagen type III mRNA than on that of procollagen type I, whilst inhibition of STAT3 exerted a stronger inhibitory effect on procollagen type III mRNA than on procollagen type I mRNA at an early time point but a stronger inhibition on procollagen type I mRNA than on procollagen type III at a later time point. Finally, the combined inhibition of ERK1/2 and STAT1 exhibited stronger suppressing effects on CF proliferation and collagen I synthesis after prolonged exposure to HG. In contrast, the combinational inhibition of ERK1/2 and STAT3 demonstrated greater inhibition on CF proliferation and collagen I synthesis at earlier time points than at later time points. These differences indicated that STAT1 and STAT3 may act on cardiac fibrosis by different mechanisms and STAT3 probably has a more complicated functional role than STAT1 in cardiac fibrosis.

In conclusion, our study indicates that like the ERK1/2 pathway, the STAT pathway in also plays an important role in the control of CF proliferation and collagen deposition in the presence of high glucose in CFs. STAT1/3 and ERK1/2 can work cooperatively to regulate cardiac fibrosis but perhaps through different mechanisms. This is the first report on the role of the STAT signaling cascade as a novel mechanism for cardiac fibrosis under simulated diabetic conditions. Our results also laid the groundwork for future development of STAT1/3 inhibitors as potential therapeutic drugs for the treatment of diabetic cardiomyopathy.

\section{References}

1 Redfield MM, Jacobsen SJ, Burnett JC Jr, Mahoney DW, Bailey KR, Rodeheffer RJ: Burden of systolic and diastolic ventricular dysfunction in the community: Appreciating the scope of the heart failure epidemic. JAMA 2003;289:194-202.

- Van Heerebeek L, Hamdani N, Handoko ML, Falcao-Pires I, Musters RJ, Kupreishvili K, Ijsselmuiden AJ, Schalkwijk CG, Bronzwaer JG, Diamant M, Borbély A, van der Velden J, Stienen GJ, Laarman GJ, Niessen HW, Paulus WJ: Diastolic stiffness of the failing diabetic heart: Importance of fibrosis, advanced glycation end products, and myocyte resting tension. Circulation 2008;117:43-51.

-3 Suskin N, McKelvie RS, Burns RJ, Latini R, Pericak D, Probstfield J, Rouleau JL, Sigouin C, Solymoss CB, Tsuyuki R, White M, Yusuf S: Glucose and insulin abnormalities relate to functional capacity in patients with congestive heart failure. Eur Heart J 2000;21:1368-1375.

-4 Jin D, Takai S, Sugiyama T, Hayashi T, Fukumoto M, Oku H, Kitaura Y, Ikeda T, Miyazaki M: Long-term angiotensin II blockade may improve not only hyperglycemia but also age-associated cardiac fibrosis. J Pharmacol Sci 2009;109:275-284.

5 Asbun J, Manso AM, Villarreal FJ: Profibrotic influence of high glucose concentration on cardiac fibroblast functions: Effects of losartan and vitamin E. Am J Physiol Heart Circ Physiol 2005;288:H227-234.

Tokudome T, Horio T, Yoshihara F, Suga S, Kawano Y, Kohno M, Kangawa K: Direct effects of high glucose and insulin on protein synthesis in cultured cardiac myocytes and DNA and collagen synthesis in cardiac fibroblasts. Metabolism 2004;53:710-715. 
Dai et al.: Signaling Mechanisms for High Glucose-Induced Cardiac Fibrosis

7 Wang P, Li HW, Wang YP, Chen H, Zhang P: Effects of recombinant human relax upon proliferation of cardiac fibroblast and synthesis of collagen under high glucose condition. J Endocrinol Invest 2009;32:242-247.

8 Ihle JN: The Stat family in cytokine signaling. Curr Opin Cell Biol 2001;13:211-217.

-9 Barry SP, Townsend PA, Latchman DS, Stephanou A: Role of the JAK-STAT pathway in myocardial injury. Trends Mol Med 2007;13:82-89.

10 Mair M, Blaas L, Österreicher CH, Casanova E, Eferl R: JAK-STAT signaling in hepatic fibrosis. Front Biosci 2011;16:2794-2811.

11 Knight D, Mutsaers SE, Prêle CM: STAT3 in tissue fibrosis: is there a role in the lung? Pulm Pharmacol Ther 2011;24:193-198.

12 Matsui F, Meldrum KK: The role of the Janus kinase family/signal transducer and activator of transcription signaling pathway in fibrotic renal disease. J Surg Res 2012;178:339-345.

13 Ortiz-Muñoz G, Lopez-Parra V, Lopez-Franco O, Fernandez-Vizarra P, Mallavia B, Flores C, Sanz A, Blanco J, Mezzano S, Ortiz A, Egido J, Gomez-Guerrero C: Suppressors of cytokine signaling abrogate diabetic nephropathy. J Am Soc Nephrol 2010;21:763-772.

14 Roskoski R Jr: ERK1/2 MAP kinases: structure, function, and regulation. Pharmacol Res 2012;66:105-143.

15 Tang M, Zhang W, Lin H, Jiang H, Dai H, Zhang Y: High glucose promotes the production of collagen types I and III by cardiac fibroblasts through a pathway dependent on extracellular-signal-regulated kinase 1/2. Mol Cell Biochem 2007;301:109-114.

16 Kovarik P, Mangold M, Ramsauer K, Heidari H, Steinborn R, Zotter A, Levy DE, Müller M, Decker T: Specificity of signaling by STAT1 depends on SH2 and C-terminal domains that regulate Ser727 phosphorylation, differentially affecting specific target gene expression. EMBO J 2001;20:91-100.

17 Athie MV, Flotow H, Hilyard KL, Cantrell DA: IL-12 selectively regulates STAT4 via phosphatidylinositol 3-kinase and Ras-independent signal transduction pathways. Eur J Immunol 2000;30:1425-1434.

18 Rebsamen MC, Arrighi JF, Juge-Aubry CE, Vallotton MB, Lang U: Epider2 mal growth factor induces hypertrophic responses and Stat5 activation in rat ventricular cardiomyocytes. J Mol Cell Cardiol 2000;32:599-610.

19 Ng DC, Long CS, Bogoyevitch MA: A role for the extracellular signal-regulated kinase and p38 mitogenactivated protein kinases in interleukin-1-stimulated delayed signal transducer and activator of transcription 3 activation, atrial natriuretic factor expression, and cardiac myocyte morphology. J Biol Chem 2001;276:29490-29498.

20 Nobori K, Munehisa Y: Intracellular signaling pathways for cardiac hypertrophy: ERK, JAK-STAT, S6 kinase. Nihon Rinsho 2007;65:196-200.

21 Fischer P, Hilfiker-Kleiner D: Survival pathways in hypertrophy and heart failure: the gp130-STAT axis. Basic Res Cardiol 2007;102:393-411.

-22 Frias MA, Rebsamen MC, Gerber-Wich, Lang U: Prostaglandin E2 activates Stat3 in neonatal rat ventricular cardiomyocytes. A role in cardiac hypertrophy. Cardiovasc Res 2007;73:57-65.

-23 Siddiquee K, Zhang S, Guida WC, Blaskovich MA, Greedy B, Lawrence HR, Yip ML, Jove R, McLaughlin MM, Lawrence NJ, Sebti SM, Turkson J: Selective chemical probe inhibitor of Stat3, identified through structurebased virtual screening, induces antitumor activity. Proc Natl Acad Sci USA 2007;104:7391-7396.

24 Livak KJ, Schmittgen TD: Analysis of relative gene expression data using real-time quantitative PCR and the 2(-Delta Delta C(T)). Method Methods 2001;25:402-408.

25 De Souza RR: Aging of myocardial collagen. Biogerontology 2002;3:325-335.

26 Beltrami CA, Finato N, Rocco M, Feruglio GA, Puricelli C, Cigola E, Quaini F, Sonnenblick EH, Olivetti G, Anversa P: Structural basis of end-stage failure in ischemic cardiomyopathy in humans. Circulation 1994;89:151-163.

27 Khan R, Sheppard R: Fibrosis in heart disease. Understanding the role of transforming growth factor-beta in cardiomyopathy, valvular disease and arrhythmia. Immunology 2006;118:10-24.

28 Mizushige K, Yao L, Noma T, Kiyomoto H, Yu Y, Hosomi N, Ohmori K, Matsuo H: Alteration in left ventricular diastolic filling and accumulation of myocardial collagen at insulin-resistant prediabetic stage of a type II diabetic rat model. Circulation 2000;101:899-907.

29 Singh VP, Baker KM, Kumar R: Activation of the intracellular renin- angiotensin system in cardiac fibroblasts by high glucose: Role in extracellular matrix production. Am J Physiol Heart Circ Physiol 2008;294:H1675-1684. 
30 Levy DE, Darnell JE Jr: Stats: transcriptional control and biological impact. Nat Rev Mol Cell Biol 2002;3:651-662.

-31 Kodama H, Fukuda K, Pan J, Makino S, Baba A, Hori S, Ogawa S: Leukemia inhibitory factor, a potent cardiac hypertrophic cytokine, activates the JAK/STAT pathway in rat cardiomyocytes. Circ Res 1997;81:656-663.

-32 Mir SA, Chatterjee A, Mitra A, Pathak K, Mahata SK, Sarkar S: Inhibition of signal transducer and activator of transcription 3 (STAT3) attenuates interleukin-6 (IL-6)-induced collagen synthesis and resultant hypertrophy in rat heart. J Biol Chem 2012;287:2666-2677.

-33 Pan J, Fukuda K, Saito M, Matsuzaki J, Kodama H, Sano M, Takahashi T, Kato T, Ogawa S: Mechanical stretch activates the JAK/STAT pathway in rat cardiomyocytes. Circ Res 1999;84:1127-1136.

34 Lee MS, Kwon HJ, Kim HS: Macrophages from nonobese diabetic mouse have a selective defect in IFN- $\gamma$ but not IFN- $\alpha / \beta$ receptor pathway. J Clin Immunol 2012;32:753-761.

35 Mineshiba J, Myokai F, Mineshiba F, Matsuura K, Nishimura F, Takashiba S: Transcriptional regulation of beta-defensin-2 by lipopolysaccharide in cultured human cervi- cal carcinoma (HeLa) cells. FEMS Immunol Med Microbiol 2005;45:37-44.

-36 Lan CC, Wu CS, Huang SM, Kuo HY, Wu IH, Liang CW, Chen GS: High-glucose environment reduces human $\beta$-defensin-2 expression in human keratinocytes: implications for poor diabetic wound healing. $\mathrm{Br}$ Dermatol 2012;166:1221-1229.

37 Martin ML, Blaxall BC: Cardiac intercellular communication: are myocytes and fibroblasts fair-weather friends? J Cardiovasc Transl Res 2012;5:768-782.

-38 Tian ZJ, Cui W, Li YJ, Hao YM, Du J, Liu F, Zhang H, Zu XG, Liu SY, Chen L, An W: Different contributions of STAT3, ERK1/2, and PI3-K signaling to cardiomyocyte hypertrophy by cardiotrophin-1. Acta Pharmacol Sin 2004;25:1157-1164.

-39 Watanabe K, Sukumaran V, Veeraveedu PT, Thandavarayan RA, Gurusamy N, Ma M, Arozal W, Sari FR, Lakshmanan AP, Arumugam S, Soetikno V, Rajavel V, Suzuki K: Regulation of inflammation and myocardial fibrosis in experimental autoimmune myocarditis. Inflamm Allergy Drug Targets 2011;10:218-225.

-40 Azar ST, Tamim H, Beyhum HN, Habbal MZ, Almawi WY: Type I (Insulin-Dependent) Diabetes Is a Th1- and Th2-Mediated Autoimmune Disease. Clin Diagn Lab Immunol 1999;6:306-310.

-41 Caffarelli C, Cavagni G, Pierdomenico R, Chiari G, Spattini A, Vanelli M: Coexistence of IgE-mediated allergy and type 1 diabetes in childhood. Int Arch Allergy Immunol 2004;134:288-294.

42 Kasraie S, Niebuhr M, Werfel T: Interleukin (IL)-31 activates signal transducer and activator of transcription (STAT)-1, STAT-5 and extracellular signal-regulated kinase 1/2 and down-regulates IL-12p40 production in activated human macrophages. Allergy 2013;68:739-747.

43 Kasraie S, Niebuhr M, Baumert K, Werfel T: Functional effects of interleukin 31 in human primary keratinocytes. Allergy 2011;66:845-852.

44 Raffaghello L, Bianchi G, Pistoia V : Immunosuppressive treatments in acute myocardial infarction and stroke.Curr Pharm Biotechnol 2012;13:59-67.

45 Barry SP, Paul A Townsend, David S Latchman, Stephanou A: Role of the JAK-STAT pathway in myocardial injury. Trends Mol Med 2006:2:82-89.

-46 McCormick J, Suleman N, Scarabelli TM, Knight RA, Latchman DS, Stephanou A: STAT1 deficiency in the heart protects against myocardial infarction by enhancing autophagy. J Cell Mol Med 2012;16:386-393.

47 Luan HF, Zhao ZB, Zhao QH, Zhu P, Xiu MY, Ji Y: Hydrogen sulfide postconditioning protects isolated rat hearts against ischemia and reperfusion injury mediated by the JAK2/STAT3 survival pathway. Braz J Med Biol Res 2012;45:898-905.

48 Matsui H, Yokoyama T, Tanaka C, Sunaga H, Koitabashi N, Takizawa T, Arai M, Kurabayashi M: Pressure mediated hypertrophy and mechanical stretch up-regulate expression of the long form of leptin receptor (ob-Rb) in rat cardiac myocytes. BMC Cell Biol 2012;13:37.

-49 Huang C, Gu H, Zhang W, Manukyan MC, Shou W, Wang M: SDF-1/CXCR4 mediates acute protection of cardiac function through myocardial STAT3 signaling following global ischemia/reperfusion injury. Am J Physiol Heart Circ Physiol 2011;301:H1496-1505.

50 Mir SA, Chatterjee A, Mitra A, Pathak K, Mahata SK, Sarkar S: Inhibition of Signal Transducer and Activator of Transcription 3 (STAT3) Attenuates Interleukin-6 (IL-6)-induced Collagen Synthesis and Resultant Hypertrophy in Rat Heart. J Biol Chem 2012;287:2666-2677. 